\title{
Cerebrovascular Manifestations of Adult-Onset Varicella Zoster Virus Infection in the Central Nervous System: A Literature Review
}

\author{
Hangfei Wu \\ Changhai Hospital \\ Ruoru Wang \\ Changhai Hospital \\ Yuanyuan Li \\ Changhai Hospital \\ Xu Sun \\ Changhai Hospital \\ Jiasi Li \\ Changhai Hospital \\ Xiaoying Bi ( $\sim$ bixiaoying2013@163.com ) \\ Changhai Hospital https://orcid.org/0000-0001-5784-7294
}

\section{Research}

Keywords: varicella-zoster virus encephalitis, ischemic stroke, cerebral infarction, intracerebral hemorrhage, venous sinus thrombosis, vasculitis, high resolution magnetic resonance

Posted Date: August 5th, 2021

DOI: https://doi.org/10.21203/rs.3.rs-762970/v1

License: (c) (i) This work is licensed under a Creative Commons Attribution 4.0 International License. Read Full License 


\section{Abstract \\ Background}

Cerebrovascular complications after adult-onset varicella-zoster virus (VZV) infection have been increasingly recognized. The aim of this study was to analyze clinical and neuroimaging findings, treatment and outcome of these patients.

\section{Methods}

Systematic literature review from January 2000 to December 2019.

\section{Results}

We analyzed 31 articles with a total of adult-onset 34 cases, including 25 (73.53\%) cases of ischemic stroke (median age 52 years), 6 of intracerebral hemorrhage (median age 70.5 years) and 3 with venous sinus thrombosis. The incidence in men was higher than in women in ischemia (72\% and $28 \%$ ) or venous sinus thrombosis groups ( $100 \%$ for men). There was median time with 42.8 days from herpes zoster infection to hospital in patients with ischemic stroke. Cognitive impairment was the most common symptoms either in the ischemic group (56\%) or hemorrhagic group (83.33\%). The lesions after VZVassociated cerebral infarction or hemorrhage were mutifocal and was most common in the parietal lobe. Venous sinus thrombosis was common in the transverse sinus (100\%). Lesions in large vessels (48\%) were common, followed by small vessels (36\%) in ischemic group and multiple beaded stenosis (16.67\%) were showed in hemorrhagic group by digital subtraction angiography or magnetic resonance angiography. $60.87 \%$ of the patients with antiviral treatment in the ischemic group had favorable prognosis. All patients with anticoagulant therapy in venous sinus thrombosis group improved well (100\%), however, $60 \%$ of the patients with intracerebral hemorrhage had a poor prognosis or died.

\section{Conclusion}

We found ischemic stroke related with VZV encephalitis is common and mainly affects the middle-aged. In general, the young patients with venous sinus thrombosis improve completely, however, the old patients with intracerebral hemorrhage have poor prognosis. When the patient represents with some neurological symptoms within 2 months after VZV infection, and multiple lesions probably induced by vasculitis showed in neuroimaging, cerebral complications related with VZV infection should be considered even though the existence of some vascular risk factors for atherosclerosis.

\section{Background}

The varicella-zoster virus (VZV) is a double-stranded DNA neurotropic alpha-herpesvirus belonging to human herpesvirus type 3. After the initial infection with chickenpox, the virus may retrograde to the sensory neuron body of the ganglion through replicating $T$ cell toxemia, thus forming a latent infection ${ }^{[1]}$. When virus replication is reactivated, it can reach the skin via anterograde axon transport, causing herpes zoster. Activated VZV is also one of the important causes of acute viral encephalitis. It has been reported herpes simplex virus (HSV) accounts for $50-75 \%$ of confirmed cases of viral encephalitis, and VZV and enteroviruses account for the majority of the remaining cases ${ }^{[2]}$.

VZV can spread to the arteries of the central nervous system(CNS), eventually leading to bleeding or ischemic complications ${ }^{[3]}$. Baudouin et al. first identified stroke associated with VZV in 1896. A study consisting of pediatric patients showed the mortality rate of cerebrovascular diseases associated with VZV infection is as high as $35 \%$ in the $1970 \mathrm{~s}^{[4]}$. In a population-based study, the stroke risk of older adults with herpes zoster infection within 3 months was reportedly increased 1.53 -fold ${ }^{[5]}$.

In recent years, with the increasing number of infective cerebrovascular lesions, it has been gradually recognized that the reactivation of VZV is associated with subsequent cerebral hemorrhage or infarction. However, most of the researches are case reports with only several cases or involved with children. Here, we review the literature with a total of adult-onset 34 cases to demonstrate clinical presentations, imaging features, possible pathogenesis, treatment and outcome in VZV-related cerebral vascular lesions. It may be helpful for early recognition, accurate diagnosis and therapeutic options.

\section{Methods}

\section{literature search and selection}

We performed a literature search to identify all published cases of cerebral vascular manifestations of VZV from January 2000 to December 2019 using MEDLINE/PubMed, Web of Science. There were no language restrictions. The case reports of children and not getting full text articles were excluded. Search terms used were "varicella-zoster virus," "encephalitis," "meningitis," or "meningoencephalitis" and one of the following terms: "ischemia," "infarction," "stroke," "hemorrhage," "venous sinus thrombosis," or "vasculopathy." We reviewed full text and additional cases were identified by reviewing the reference section of the retrieved articles. Each article was evaluated by two independently investigators to determine inclusion in the final review.

All the patients met the diagnostic criteria reported as follows ${ }^{[6,7]}$ : (1) VZV vascular lesions (ischemic stroke, hemorrhage, venous sinus thrombosis, or vasculitis) were confirmed by imaging findings of computed tomography (CT), magnetic resonance imaging (MRI), magnetic resonance angiography 
(MRV), or digital subtraction angiography (DSA), (2) cerebrospinal fluid(CSF) results of VZV infection were confirmed according to diagnostic criteria by a consensus article ${ }^{[8]}$, and (3) exclusion of other causes for cerebral vascular disease.

\section{Data extraction}

Two investigators collected data from the selected articles. The following information were extracted: last name of the first author, demographics, clinical symptoms, etiology, CSF examination, time from onset of Herpes zoster infection to hospital, imaging data, therapy and outcome. Clinical symptoms include headache, fever, cognitive abnormalities, hemiplegia, peripheral facial palsy, aphasia, seizure or ataxia. Diagnostic tests of CNS VZV infection were polymerase chain reaction (PCR), anti-VZV IgG antibody and viral culture. Cerebrovascular lesions were identified by CT, MRI or MRV. Large and small vessels, and evidence of vasculitis were assessed by DSA or MRA. The Large arteries were the internal carotid artery, the anterior cerebral artery and the middle cerebral artery in the anterior circulation, and the posterior cerebral artery, the vertebral artery and basilar artery in the posterior circulation. Small vessels included the small penetrating arteries. We also recorded the use of antiviral therapy, corticosteroids and anti-platelet or anticoagulant medication. In clinical trials, the Modified Rankin Scale (mRS) was commonly used scales to assess outcome. The definition of poor outcome was mRS greater than or equal to 3 points while good outcome defined as a mRS score of $0-2$ points ${ }^{[9]}$.

All statistical analyses were performed using SPSS 19.0 (IBM Corp., Armonk, NY, USA).

\section{Results}

\section{Systematic review}

From January 2000 to December 2019, through a preliminary electronic literature search and manual literature search, we finally analyzed 31 articles with a total of 34 cases. There were 25 cases(25/34, 73.53\%) with ischemic stroke ${ }^{[10-32]}, 6$ cases(6/34, 17.65\%) with intracerebral hemorrhage ${ }^{[33-38]}$, and 3 patients(3/34, 8.82\%) with venous sinus thrombosis ${ }^{[39-41]}$.

\section{Ischemic Stroke}

The age of onset varied between 24 and 85 years old with a median age of 52 . Eighteen patients were males (18/25, 72\%) with an $18: 7$ of male to female ratio. $40 \%(10 / 25)$ of the patients had no previous history of varicella zoster infection. $40 \%(10 / 25)$ of the patients developed ischemic manifestations within one month after zoster infection, while $12 \%(3 / 25)$ of the patients demonstrated ischemic symptoms within $2-3$ months. The longest interval of onset was seven months after the existence of zoster with a median time of 42.8 days. Initial clinical presentations of patients included cognitive impairment $(14 / 25,56 \%)$, lalopathy $(10 / 25,40 \%)$, headache $(9 / 25,36 \%)$, hemiplegia $(8 / 25,32 \%)$, fever $(6 / 25,24 \%)$, ataxia $(4 / 25,16 \%)$, epilepsy $(4 / 25$, $16 \%)$ and peripheral facial paralysis $(3 / 25,12 \%)$.

CSF VZV-DNA positive was confirmed by PCR in 10 patients (10/25, 40\%), and there were 9 cases (9/25, 36\%) with positive anti-VZV IgG in CSF test. Both VZV DNA and anti-VZV IgG in CSF were positive in 5 cases $(5 / 25,20 \%)$. Anti-VZV IgG positive for both VZV CSF and serum was in one case (1/25, $4 \%$ ). VZV was confirmed in one patient by viral culture. Multiple ischemic lesions were found in $52 \%(13 / 25)$ distributed most commonly in both anterior and posterior circulations simultaneously, which is different from cerebrovascular disease caused by common atherosclerosis. The parietal and occipital lobes, as well as brainstem were the main sites of ischemic stroke after VZV infection. The remaining locations were basal ganglia, temporal and frontal lobes and cerebellum in sequence. Evidence of vasculitis was found in $40 \%$ (10/25) of patients. Large vessel lesions were found in $48 \%$ (12/25) patients, and small vessel lesions in $36 \%(9 / 25)$ on MRA or DSA images.

All patients $(100 \%, 24 / 24)$ received Intravenous acyclovir at the early stage except one patient whose treatment was not mentioned, among them $60.87 \%$ $(14 / 23)$ (not mention of prognosis in one patient) had $0-2 \mathrm{mRs}$. There was no significant difference in either mortality (22.22\% vs $21.43 \%)$ or favorable prognosis $(77.78 \%$ vs $50 \%$ ) between antiviral drug therapy alone and antiviral drug combined with steroid. One patient recovered well on the combination of antiviral, steroid and anti-platelet treatment.

Demographics, clinical features, imaging abnormalities, and outcome were presented separately for patients with ischemic stroke in Table 1. The characteristics of the included cases were presented in Table 2. 
Table 1

Demographics, clinical features, imaging abnormalities, and outcome are presented for patients with ischemic stroke

\begin{tabular}{|c|c|}
\hline \multicolumn{2}{|l|}{ Demographics } \\
\hline$n$ & 25 \\
\hline Median age, years & $52(24-85)$ \\
\hline Male gender & $72 \%(18 / 25)$ \\
\hline Days from Herpes zoster infection to the occurrence of neurologic symptoms (median), $n=15$ & $42.8(4-210)$ \\
\hline \multicolumn{2}{|l|}{ Clinical features } \\
\hline Headache & $36 \%(9 / 25)$ \\
\hline Fever & $24 \%(6 / 25)$ \\
\hline Cognitive impairment & $56 \%(14 / 25)$ \\
\hline Hemiplegia & $32 \%(8 / 25)$ \\
\hline Peripheral facial palsy & $12 \%(3 / 25)$ \\
\hline Lalopathy (aphasia or dysarthria) & $40 \%(10 / 25)$ \\
\hline Seizure & $16 \%(4 / 25)$ \\
\hline Ataxia & $16 \%(4 / 25)$ \\
\hline \multicolumn{2}{|l|}{ Diagnostic testing } \\
\hline PCR positive for VZV(CSF) only & $40 \%(10 / 25)$ \\
\hline anti-VZV IgG positive for VZV(CSF) only & $36 \%(9 / 25)$ \\
\hline Both PCR and anti-VZV IgG positive for VZV(CSF) & $20 \%(5 / 25)$ \\
\hline Both anti-VZV IgG positive for VZV CSF and serum & $4 \%(1 / 25)$ \\
\hline Viral culture positive for VZV(CSF) & $4 \%(1 / 25)$ \\
\hline \multicolumn{2}{|l|}{ Neuroimaging } \\
\hline Evidence for vasculitis & $40 \%(10 / 25)$ \\
\hline \multicolumn{2}{|l|}{ Affected vessels } \\
\hline Small-sized & $36 \%(9 / 25)$ \\
\hline Large-sized & $48 \%(12 / 25)$ \\
\hline Not done & $16 \%(4 / 25)$ \\
\hline \multicolumn{2}{|l|}{ Affected areas of circulation } \\
\hline Anterior & $36 \%(9 / 25)$ \\
\hline Posterior & $24 \%(6 / 25)$ \\
\hline Mixed & $40 \%(10 / 25)$ \\
\hline \multicolumn{2}{|l|}{ Distribution of lesions } \\
\hline Single & $48 \%(12 / 25)$ \\
\hline Multiple & $52 \%(13 / 25)$ \\
\hline \multicolumn{2}{|l|}{ Treatment } \\
\hline Acyclovir treatment & $100 \%(24 / 24)$ \\
\hline Steroid treatment & $62.50 \%(15 / 24)$ \\
\hline Anti-platelet medication & $4.17 \%(1 / 24)$ \\
\hline \multicolumn{2}{|l|}{ Outcome } \\
\hline Good outcome (mRS 0-2) & $60.87 \%(14 / 23)$ \\
\hline Unfavorable outcome (mRS 3-5) & $17.39 \%(4 / 23)$ \\
\hline Death & $21.74 \%(5 / 23)$ \\
\hline
\end{tabular}


Table 2

The characteristics of twenty-five ischemic stroke cases

\begin{tabular}{|c|c|c|c|c|c|c|c|c|}
\hline Case & Reference & $\begin{array}{l}\text { Sex, } \\
\text { age(years) }\end{array}$ & $\begin{array}{l}\text { Clinical } \\
\text { features }\end{array}$ & Diagnosis test & $\begin{array}{l}\text { Time from } \\
\text { Herpes } \\
\text { zoster } \\
\text { infection }\end{array}$ & $\begin{array}{l}\text { Affected brain } \\
\text { region }\end{array}$ & Treatment & Outcome (mRS) \\
\hline 1 & $\begin{array}{l}\text { David } \\
\text { et al.2015 }\end{array}$ & Male,51 & $\begin{array}{l}\text { Fever, chills, } \\
\text { confusion } \\
\text { herpes zoster, } \\
\text { lethargic } \\
\text { disoriented }\end{array}$ & $\begin{array}{l}\text { CSF, anti-VZV } \\
\operatorname{lgG}(+)\end{array}$ & 2 weeks & $\begin{array}{l}\text { Bilateral gray- } \\
\text { white matter }\end{array}$ & $\begin{array}{l}\text { acyclovir } \\
\text { Dexamethasone }\end{array}$ & $\begin{array}{l}\text { Complete } \\
\text { recovery }(0)\end{array}$ \\
\hline 2 & $\begin{array}{l}\text { Jeroen } \\
\text { et al.2014 }\end{array}$ & Male,72 & $\begin{array}{l}\text { Herpes zoster, } \\
\text { cognitive } \\
\text { abnormalities, } \\
\text { the left-sided } \\
\text { facial paresis, } \\
\text { difficulty } \\
\text { retrieving some } \\
\text { words, }\end{array}$ & $\begin{array}{l}\text { CSF, PCR } \\
\text { VZV }(+)\end{array}$ & 6 weeks & $\begin{array}{l}\text { Right internal } \\
\text { capsule }\end{array}$ & Acyclovir & $\begin{array}{l}\text { Paresis of the } \\
\text { left arm and } \\
\text { left-sided facial } \\
\text { paresis(1) }\end{array}$ \\
\hline 3 & $\begin{array}{l}\text { Tiago } \\
\text { et al.2014 }\end{array}$ & Male,72 & $\begin{array}{l}\text { Headache, } \\
\text { anorexia, } \\
\text { nausea, zoster, } \\
\text { memory deficit, } \\
\text { incoherent } \\
\text { speech }\end{array}$ & $\begin{array}{l}\text { CSF, PCR } \\
\text { VZV }(+)\end{array}$ & 11 days & $\begin{array}{l}\text { Posterior } \\
\text { ischemic optic } \\
\text { neuropathy }\end{array}$ & $\begin{array}{l}\text { Prednisolone } \\
\text { Acyclovir }\end{array}$ & Not reported \\
\hline 4 & $\begin{array}{l}\text { Francisco } \\
\text { et al.2014 }\end{array}$ & Male,26 & $\begin{array}{l}\text { Zoster, VZV } \\
\text { meningitis }\end{array}$ & $\begin{array}{l}\text { CSF, PCR } \\
\text { VZV }(+)\end{array}$ & 4 months & $\begin{array}{l}\text { Right occipital } \\
\text { lobe }\end{array}$ & Not reported & Not reported \\
\hline 5 & $\begin{array}{l}\text { Francesca } \\
\text { et al.2014 }\end{array}$ & Female,67 & $\begin{array}{l}\text { Rash, left } \\
\text { peripheral } \\
\text { facial palsy, } \\
\text { gait instability, } \\
\text { cerebellar } \\
\text { ataxia }\end{array}$ & $\begin{array}{l}\text { CSF,PCR(+)and } \\
\text { anti-VZV IgG in } \\
\text { CSF }\end{array}$ & 2 weeks & $\begin{array}{l}\text { Left pons, left } \\
\text { midbrain, right } \\
\text { periventricular } \\
\text { area }\end{array}$ & $\begin{array}{l}\text { Acyclovir } \\
\text { Carbamazepine }\end{array}$ & $\begin{array}{l}\text { Complete } \\
\text { recovery }(0)\end{array}$ \\
\hline 6 & $\begin{array}{l}\text { Brian } \\
\text { et al.2012 }\end{array}$ & Male,69 & $\begin{array}{l}\text { Vision loss, } \\
\text { word-finding } \\
\text { difficulties, } \\
\text { dysarthria, } \\
\text { short-term } \\
\text { memory loss, } \\
\text { ataxia, } \\
\text { dizziness } \\
\text { expressive } \\
\text { language } \\
\text { difficulties, } \\
\text { unable to } \\
\text { identify any } \\
\text { elements of } \\
\text { date or location }\end{array}$ & $\begin{array}{l}\text { CSF, anti-VZV } \\
\operatorname{lgG}(+)\end{array}$ & $\begin{array}{l}\text { No history } \\
\text { of zoster } \\
\text { experienced }\end{array}$ & $\begin{array}{l}\text { Left corona } \\
\text { radiata, basal } \\
\text { ganglia ,right } \\
\text { basal ganglia, } \\
\text { both thalam, } \\
\text { periventricular } \\
\text { white matter, } \\
\text { right superior } \\
\text { cerebellum }\end{array}$ & $\begin{array}{l}\text { Cyclophosphamide } \\
\text { Rednisone; } \\
\text { Acyclovir }\end{array}$ & $\begin{array}{l}\text { Complete } \\
\text { recovery }(0)\end{array}$ \\
\hline 7 & $\begin{array}{l}\text { Yu- } \\
\text { Miyazaki et } \\
\text { al.2008 }\end{array}$ & Male,66 & $\begin{array}{l}\text { Headache, } \\
\text { fever, and } \\
\text { altered mental } \\
\text { status, zoster } \\
\text { rash } \\
\text { consciousness } \\
\text { and stiff neck }\end{array}$ & $\begin{array}{l}\text { CSF,VZV-IgG } \\
\text { antibody and } \\
\text { VZV-DNA (+) }\end{array}$ & $\begin{array}{l}\text { Not } \\
\text { reported }\end{array}$ & $\begin{array}{l}\text { Brainstem, } \\
\text { vermis of } \\
\text { cerebellum and } \\
\text { cerebral white } \\
\text { matter }\end{array}$ & $\begin{array}{l}\text { Acyclovir } \\
\text { Methylprednisolone }\end{array}$ & Death \\
\hline 8 & $\begin{array}{l}\text { O.Outteryck } \\
\text { et al. } 2004\end{array}$ & Male,57 & $\begin{array}{l}\text { Fever, } \\
\text { headache, } \\
\text { abnormal } \\
\text { behavior, } \\
\text { mental } \\
\text { retardation }\end{array}$ & $\begin{array}{l}\text { CSF ,PCR VZV } \\
(+)\end{array}$ & $\begin{array}{l}\text { Not } \\
\text { reported }\end{array}$ & $\begin{array}{l}\text { Amygdala, } \\
\text { cerebellum, } \\
\text { brainstem, deep } \\
\text { white matter }\end{array}$ & Acyclovir & Death \\
\hline 9 & $\begin{array}{l}\text { Manuel } \\
\text { et al.2002 }\end{array}$ & Female,68 & $\begin{array}{l}\text { Mental } \\
\text { retardation, } \\
\text { difficulty } \\
\text { walking, right } \\
\text { hemiplegia, } \\
\text { aphasia, rash }\end{array}$ & $\begin{array}{l}\text { CSF ,PCR VZV } \\
(+)\end{array}$ & $\begin{array}{l}\text { Not } \\
\text { reported }\end{array}$ & $\begin{array}{l}\text { Left internal } \\
\text { capsule }\end{array}$ & Acyclovir & Death \\
\hline 10 & $\begin{array}{l}\text { Nasir } \\
\text { et al.2003 }\end{array}$ & Male,52 & $\begin{array}{l}\text { Chicken pox, } \\
\text { headache, } \\
\text { nausea, vomit, } \\
\text { and } \\
\text { photophobia, } \\
\text { confusion }\end{array}$ & $\begin{array}{l}\text { CSF, viral } \\
\text { culture VZV(+) }\end{array}$ & 5 weeks & $\begin{array}{l}\text { Left frontal, left } \\
\text { parietal, right } \\
\text { medial temporal, } \\
\text { and right } \\
\text { occipital lobes }\end{array}$ & $\begin{array}{l}\text { Ceftriaxone } \\
\text { Methylprednisolone } \\
\text { Acyclovir }\end{array}$ & $\begin{array}{l}\text { Right-side } \\
\text { hemiparesis } \\
\text { persisted(2) }\end{array}$ \\
\hline
\end{tabular}




\begin{tabular}{|c|c|c|c|c|c|c|c|c|}
\hline Case & Reference & $\begin{array}{l}\text { Sex, } \\
\text { age(years) }\end{array}$ & $\begin{array}{l}\text { Clinical } \\
\text { features }\end{array}$ & Diagnosis test & $\begin{array}{l}\text { Time from } \\
\text { Herpes } \\
\text { zoster } \\
\text { infection }\end{array}$ & $\begin{array}{l}\text { Affected brain } \\
\text { region }\end{array}$ & Treatment & Outcome (mRS) \\
\hline 11 & $\begin{array}{l}\text { John } \\
\text { et al.2012 }\end{array}$ & Male,50 & $\begin{array}{l}\text { Chicken pox, } \\
\text { seizure and } \\
\text { coma, rash, } \\
\text { deeply } \\
\text { comatose }\end{array}$ & $\begin{array}{l}\text { CSF ,PCR VZV } \\
(+)\end{array}$ & 4 days & $\begin{array}{l}\text { Posterolateral } \\
\text { medulla }\end{array}$ & $\begin{array}{l}\text { Levetiracetam } \\
\text { Acyclovir } \\
\text { Prednisone }\end{array}$ & $\begin{array}{l}\text { Awake and } \\
\text { oriented with } \\
\text { significant } \\
\text { psychomotor } \\
\text { slowing and } \\
\text { persistent } \\
\text { quadriparesis(4) }\end{array}$ \\
\hline 12 & $\begin{array}{l}\text { Mckelvie } \\
\text { et al.2002 }\end{array}$ & Female,67 & $\begin{array}{l}\text { Nausea, } \\
\text { tiredness, } \\
\text { hyperaesthesia, } \\
\text { fever, } \\
\text { disorientation } \\
\text { to time, place, } \\
\text { verbal } \\
\text { response. }\end{array}$ & $\begin{array}{l}\text { CSF,PCR VZV } \\
(+)\end{array}$ & 2 weeks & $\begin{array}{l}\text { Bilateral } \\
\text { cerebellum }\end{array}$ & $\begin{array}{l}\text { Dexamethasone } \\
\text { Acyclovir }\end{array}$ & Death \\
\hline 13 & $\begin{array}{l}\text { Katchanov } \\
\text { et al.2010 }\end{array}$ & Male,36 & $\begin{array}{l}\text { Locked-in } \\
\text { syndrome }\end{array}$ & $\begin{array}{l}\text { CSF, ,PCR VZV } \\
(+)\end{array}$ & $\begin{array}{l}\text { Not } \\
\text { reported }\end{array}$ & $\begin{array}{l}\text { Bilateral pons } \\
\text { and midbrain }\end{array}$ & $\begin{array}{l}\text { Acylovir, } \\
\text { Methylprednisolone }\end{array}$ & Severe (5) \\
\hline 14 & $\begin{array}{l}\text { Katchanov } \\
\text { et al.2010 }\end{array}$ & Male,32 & $\begin{array}{l}\text { Global aphasia, } \\
\text { right } \\
\text { hemiplegia }\end{array}$ & $\begin{array}{l}\text { CSF, VZV-IgG } \\
\text { antibody }(+)\end{array}$ & $\begin{array}{l}\text { Not } \\
\text { reported }\end{array}$ & $\begin{array}{l}\text { Left MCA } \\
\text { territory, left PCA } \\
\text { territory }\end{array}$ & $\begin{array}{l}\text { Acylovir, } \\
\text { Methylprednisolone }\end{array}$ & Severe (5) \\
\hline 15 & $\begin{array}{l}\text { Gilden } \\
\text { et al.2002 }\end{array}$ & Male,71 & $\begin{array}{l}\text { Zoster, } \\
\text { headache, mild } \\
\text { confusion, foot } \\
\text { numbness, } \\
\text { unsteady gait, } \\
\text { difficulty } \\
\text { finding words, } \\
\text { numbness of } \\
\text { the left hand, } \\
\text { weakness of } \\
\text { the left } \\
\text { leg }\end{array}$ & $\begin{array}{l}\text { CSF, VZV-IgG } \\
\text { antibody }(+)\end{array}$ & 4 weeks & $\begin{array}{l}\text { Right } \\
\text { pericallosal, } \\
\text { occlusion of the } \\
\text { anterior cerebral } \\
\text { artery on the } \\
\text { right side and } \\
\text { stenosis on the } \\
\text { left side }\end{array}$ & Acyclovir & $\begin{array}{l}\text { Complete } \\
\text { recovery }(0)\end{array}$ \\
\hline 16 & $\begin{array}{l}\text { Gilden } \\
\text { et al.2002 }\end{array}$ & Male,76 & $\begin{array}{l}\text { Zoster, } \\
\text { headache, lost } \\
\text { all vision in the } \\
\text { left eye }\end{array}$ & $\begin{array}{l}\text { CSF, VZV-IgG } \\
\text { antibody }(+)\end{array}$ & 7 months & $\begin{array}{l}\text { Posterior } \\
\text { ischemic optic } \\
\text { neuropathy }\end{array}$ & Acyclovir & $\begin{array}{l}\text { Complete } \\
\text { recovery }(0)\end{array}$ \\
\hline 17 & $\begin{array}{l}\text { Richard } \\
\text { et al.2011 }\end{array}$ & Male,80 & $\begin{array}{l}\text { zoster, sudden } \\
\text { painless loss } \\
\text { of vision in the } \\
\text { left eye }\end{array}$ & $\begin{array}{l}\text { CSF, VZV-IgG } \\
\text { and VZV-IgM } \\
\text { antibody }(+)\end{array}$ & One month & $\begin{array}{l}\text { Occlusion of the } \\
\text { left ophthalmic } \\
\text { artery }\end{array}$ & $\begin{array}{l}\text { Methylprednisolone, } \\
\text { Prednisone } \\
\text { Acyclovir }\end{array}$ & $\begin{array}{l}\text { Visual acuity } \\
\text { had improved to } \\
20 / 50(2)\end{array}$ \\
\hline 18 & $\begin{array}{l}\text { Andreas } \\
\text { et al.2002 }\end{array}$ & Male,28 & $\begin{array}{l}\text { HIV+,HCV+, } \\
\text { progressive } \\
\text { dizziness, left- } \\
\text { side weakness }\end{array}$ & $\begin{array}{l}\text { CSF, VZV DNA } \\
\text { and IgG } \\
\text { antibody }(+)\end{array}$ & $\begin{array}{l}\text { Not } \\
\text { reported }\end{array}$ & $\begin{array}{l}\text { Right } \\
\text { occipitotemporal, } \\
\text { parieto-occipital } \\
\text { cortices }\end{array}$ & Acyclovir & $\begin{array}{l}\text { Complete } \\
\text { recovery }(0)\end{array}$ \\
\hline 19 & $\begin{array}{l}\text { Andrew } \\
\text { et al.2003 }\end{array}$ & Female,51 & $\begin{array}{l}\text { Confusion, } \\
\text { disorientation, } \\
\text { vertigo, } \\
\text { decreased right } \\
\text { hearing, right- } \\
\text { sided } \\
\text { clumsiness left } \\
\text { leg weakness, } \\
\text { seizure, }\end{array}$ & $\begin{array}{l}\text { CSF, VZV IgG } \\
\text { antibody }(+)\end{array}$ & $\begin{array}{l}\text { Not } \\
\text { reported }\end{array}$ & $\begin{array}{l}\text { Right facial } \\
\text { nerve, brainstem, } \\
\text { thalamus, } \\
\text { caudate nucleus, } \\
\text { internal capsule, } \\
\text { left temporal } \\
\text { lobe,parietal and } \\
\text { occipital lobes, } \\
\text { hippocampus, } \\
\text { insula, } \\
\text { periventricular } \\
\text { white matter }\end{array}$ & $\begin{array}{l}\text { Acyclovir } \\
\text { Methylprednisolone }\end{array}$ & $\begin{array}{l}\text { Decreased } \\
\text { arousal, } \\
\text { spastic } \\
\text { quadriparesis, } \\
\text { severe } \\
\text { dysphagia, } \\
\text { tracheostomy } \\
\text { dependence(5) }\end{array}$ \\
\hline 20 & $\begin{array}{l}\text { Takeshi } \\
\text { et al.2006 }\end{array}$ & Male,36 & $\begin{array}{l}\text { HIV+, fever, } \\
\text { convulsions, } \\
\text { herpes zoster, } \\
\text { neck stiffness }\end{array}$ & $\begin{array}{l}\text { CSF, VZV DNA } \\
\text { and IgG } \\
\text { antibody(+) } \\
\text { Serum, VZV } \\
\text { IgG } \\
\text { antibody(+) }\end{array}$ & 9 days & $\begin{array}{l}\text { A stenotic lesion } \\
\text { in the left middle } \\
\text { cerebral artery, } \\
\text { higher-intensity } \\
\text { perivascular } \\
\text { areas within } \\
\text { subcortical } \\
\text { regions }\end{array}$ & $\begin{array}{l}\text { Acyclovir } \\
\text { Prednisolone }\end{array}$ & $\begin{array}{l}\text { Complete } \\
\text { recovery }(0)\end{array}$ \\
\hline
\end{tabular}




\begin{tabular}{|c|c|c|c|c|c|c|c|c|}
\hline Case & Reference & $\begin{array}{l}\text { Sex, } \\
\text { age(years) }\end{array}$ & $\begin{array}{l}\text { Clinical } \\
\text { features }\end{array}$ & Diagnosis test & $\begin{array}{l}\text { Time from } \\
\text { Herpes } \\
\text { zoster } \\
\text { infection }\end{array}$ & $\begin{array}{l}\text { Affected brain } \\
\text { region }\end{array}$ & Treatment & Outcome (mRS) \\
\hline 21 & $\begin{array}{l}\text { Deepti } \\
\text { et al.2012 }\end{array}$ & Female,48 & $\begin{array}{l}\text { HIV+, rash, } \\
\text { headache, } \\
\text { vomit, } \\
\text { numbness of } \\
\text { the left half of } \\
\text { the face and } \\
\text { right arm }\end{array}$ & $\begin{array}{l}\text { CSF, IgG } \\
\text { antibody }(+)\end{array}$ & 7 days & $\begin{array}{l}\text { Left lateral } \\
\text { medullary }\end{array}$ & $\begin{array}{l}\text { Aspirin } \\
\text { Acyclovir } \\
\text { Prednisone }\end{array}$ & $\begin{array}{l}\text { Complete } \\
\text { recovery }(0)\end{array}$ \\
\hline 22 & $\begin{array}{l}\text { Gustavo } \\
\text { et al.2008 }\end{array}$ & Female,24 & $\begin{array}{l}\text { HIV+, rash, left } \\
\text { peripheral } \\
\text { facial palsy, } \\
\text { headache and } \\
\text { mild left sided } \\
\text { weakness, } \\
\text { dysarthria, and } \\
\text { dysphagia }\end{array}$ & $\begin{array}{l}\text { CSF, VZV } \\
\text { DNA(+) }\end{array}$ & 2 weeks & $\begin{array}{l}\text { Acute ischemic } \\
\text { pontine } \\
\text { infarction } \\
\text { MRA: multiple } \\
\text { areas of } \\
\text { narrowing and } \\
\text { beading in } \\
\text { posterior and } \\
\text { anterior } \\
\text { circulation } \\
\text { DSA: segmental } \\
\text { caliber narrowing } \\
\text { in distal vertebral } \\
\text { arteries and } \\
\text { basilar artery. } \\
\text { Similar changes } \\
\text { were seen in } \\
\text { distal left } \\
\text { internal carotid } \\
\text { artery (ICA), } \\
\text { proximal left } \\
\text { middle cerebral } \\
\text { artery, and } \\
\text { proximal left } \\
\text { anterior cerebral } \\
\text { artery }\end{array}$ & $\begin{array}{l}\text { Acyclovir } \\
\text { Methylprednisolone }\end{array}$ & $\begin{array}{l}\text { Complete } \\
\text { recovery }(0)\end{array}$ \\
\hline 23 & $\begin{array}{l}\text { Kalita } \\
\text { et al.2004 }\end{array}$ & Male,27 & $\begin{array}{l}\text { Herpes zoster, } \\
\text { numbness on } \\
\text { his left arm } \\
\text { and face, left } \\
\text { hemiplegia }\end{array}$ & $\begin{array}{l}\text { CSF, IgG and } \\
\text { IgM } \\
\text { antibody(+) }\end{array}$ & 3 months & $\begin{array}{l}\text { Right middle } \\
\text { cerebral arterial } \\
\text { territory, } \\
\text { complete } \\
\text { occlusion of the } \\
\text { right MCA }\end{array}$ & Acyclovir & $\begin{array}{l}\text { Complete } \\
\text { recovery }(0)\end{array}$ \\
\hline 24 & $\begin{array}{l}\text { Jyotsna } \\
\text { et al.2011 }\end{array}$ & Male,35 & $\begin{array}{l}\text { HIV+, left-sided } \\
\text { body weakness } \\
\text { and slurred } \\
\text { speech }\end{array}$ & $\begin{array}{l}\text { CSF, VZV } \\
\text { DNA(+) }\end{array}$ & $\begin{array}{l}\text { Not } \\
\text { reported }\end{array}$ & $\begin{array}{l}\text { An acute right } \\
\text { basal ganglia } \\
\text { infarct } \\
\text { Bilateral carotid } \\
\text { arteries revealed } \\
\text { occlusion }\end{array}$ & Acyclovir & $\begin{array}{l}\text { Minimal } \\
\text { residual } \\
\text { weakness(1) }\end{array}$ \\
\hline 25 & $\begin{array}{l}\text { Eleonora } \\
\text { et al.2008 }\end{array}$ & Female,85 & $\begin{array}{l}\text { Headache, mild } \\
\text { hyperthermia, } \\
\text { confusion and } \\
\text { seizures }\end{array}$ & $\begin{array}{l}\text { CSF, VZV DNA } \\
\text { and IgG } \\
\text { antibody }(+)\end{array}$ & $\begin{array}{l}\text { Not } \\
\text { reported }\end{array}$ & $\begin{array}{l}\text { Right superior } \\
\text { temporal gyrus, } \\
\text { left paramedian } \\
\text { parieto-occipital } \\
\text { cortex }\end{array}$ & $\begin{array}{l}\text { Acyclovir } \\
\text { Methylprednisolone }\end{array}$ & Death \\
\hline
\end{tabular}

\section{Intracerebral Hemorrhage}

The age of onset varied between 45 and 79 years old with a median age of 70.5 . The sex ratio of the patients is $1: 1$. One-third of the patients had a history of immunosuppression use. Clinical presentations of patients included cognitive impairment $(5 / 6,83.33 \%)$, headache (4/6, 66.67\%), hemiplegia (4/6, $66.67 \%)$ and fever $(4 / 6,66.67 \%)$.

Multifocal cerebral hemorrhage was found in $66.67 \%$ located in parietal lobe (3/6,50\%), occipital lobe (2/6, 33.33\%), temporal lobe $(2 / 6,33.33 \%)$, frontal lobe $(2 / 6,33.33 \%)$, cerebellum $(1 / 6,16.67 \%)$ and brainstem $(1 / 6,16.67 \%)$. There were two patients with ventricular and subarachnoid hemorrhage. Evidence of vasculitis was found in $16.67 \%$ (1/6) of patients. DSA and MRA examinations revealed multiple beaded stenosis in both the anterior and posterior circulation vessels.

$100 \%(5 / 5)$ of the patients received antiviral therapy, and among them $40 \%(2 / 5)$ had used steroid at the early stage of the disease. However, $60 \%(3 / 5)$ of the patents had a poor prognosis or died (not mention of therapy and prognosis in one patient).

Demographics, clinical features, imaging abnormalities, and outcome were presented separately for patients with intracerebral hemorrhage in Table 3. The characteristics of the included cases were presented in Table 4. 
Table 3

Demographics, clinical features, imaging abnormalities, and outcome are presented for patients with intracerebral hemorrhage

\begin{tabular}{|c|c|}
\hline \multicolumn{2}{|l|}{ Demographics } \\
\hline$n$ & 6 \\
\hline Median age (IQR), years & $70.5(45-79)$ \\
\hline Male gender & $50 \%(3 / 6)$ \\
\hline Immunosuppression & $33.33 \%(2 / 6)$ \\
\hline \multicolumn{2}{|l|}{ Clinical features } \\
\hline Headache & $66.67 \%(4 / 6)$ \\
\hline Fever & $66.67 \%(4 / 6)$ \\
\hline Cognitive impairment & $83.33 \%(5 / 6)$ \\
\hline Hemiplegia & $66.67 \%(4 / 6)$ \\
\hline \multicolumn{2}{|l|}{ Diagnostic testing } \\
\hline PCR positive for VZV(CSF) only & $33.33 \%(2 / 6)$ \\
\hline anti-VZV IgG positive for VZV(CSF) only & $16.67 \%(1 / 6)$ \\
\hline Both PCR and anti-VZV IgG positive for VZV(CSF) & $16.67 \%(1 / 6)$ \\
\hline Both anti-VZV IgG positive for VZV CSF and serum & $33.33 \%(2 / 6)$ \\
\hline \multicolumn{2}{|l|}{ Neuroimaging } \\
\hline Evidence for vasculitis & $16.67 \%(1 / 6)$ \\
\hline \multicolumn{2}{|l|}{ Affected areas of circulation } \\
\hline Anterior & $0 \%(0 / 6)$ \\
\hline Posterior & $16.67 \%(1 / 6)$ \\
\hline Both anterior and posterior & $83.33 \%(5 / 6)$ \\
\hline \multicolumn{2}{|l|}{ Distribution of lesions } \\
\hline Single & $33.33 \%(2 / 6)$ \\
\hline Multiple & $66.67 \%(4 / 6)$ \\
\hline \multicolumn{2}{|l|}{ Treatment } \\
\hline Acyclovir treatment & $100 \%(5 / 5)$ \\
\hline Steroid treatment & $40 \%(2 / 5)$ \\
\hline \multicolumn{2}{|l|}{ Outcome } \\
\hline Good outcome (mRS 0-2) & $40 \%(2 / 5)$ \\
\hline Unfavorable outcome (mRS 3-5) & $0 \%(0 / 5)$ \\
\hline Death & $60 \%(3 / 5)$ \\
\hline
\end{tabular}


Table 4

The characteristics of six intracerebral hemorrhage cases

\begin{tabular}{|c|c|c|c|c|c|c|c|c|}
\hline Case & Reference & $\begin{array}{l}\text { Sex, } \\
\text { age(years) }\end{array}$ & $\begin{array}{l}\text { Immuno- } \\
\text { suppression }\end{array}$ & Clinical features & $\begin{array}{l}\text { Diagnosis } \\
\text { test }\end{array}$ & Affected brain region & Treatment & $\begin{array}{l}\text { Outcome } \\
\text { (mRS) }\end{array}$ \\
\hline 1 & $\begin{array}{l}\text { Inés } \\
\text { et al.2014 }\end{array}$ & Female,45 & Yes & $\begin{array}{l}\text { Headache, } \\
\text { hemianopia, } \\
\text { nausea, vomi, } \\
\text { right brachiocrural } \\
\text { hemiparesis, rash }\end{array}$ & $\begin{array}{l}\text { CSF and } \\
\text { serum, } \\
\text { anti-VZV } \\
\operatorname{lgG}(+)\end{array}$ & $\begin{array}{l}\text { Hemorrhage in the } \\
\text { occipital and right parietal } \\
\text { lobes associated with a } \\
\text { left frontal subarachnoid } \\
\text { hemorrhage }\end{array}$ & Acyclovir & NIHSS 2(2) \\
\hline 2 & $\begin{array}{l}\text { Wonki } \\
\text { et al.2012 }\end{array}$ & Female,66 & Yes & $\begin{array}{l}\text { Headache, fever, } \\
\text { papules, } \\
\text { confusional } \\
\text { mental state }\end{array}$ & $\begin{array}{l}\text { CSF , anti- } \\
\text { VZV } \\
\operatorname{lgM}(+)\end{array}$ & $\begin{array}{l}\text { Hemorrhage in the left } \\
\text { midbrain region, left } \\
\text { ventral pons and midbrain } \\
\text { extending to the } \\
\text { contralateral medial } \\
\text { temporal lobe }\end{array}$ & $\begin{array}{l}\text { Acyclovir } \\
\text { Antibiotics }\end{array}$ & Death \\
\hline 3 & $\begin{array}{l}\text { Kazuya } \\
\text { et al.2015 }\end{array}$ & Male,75 & No & Stupor & $\begin{array}{l}\text { CSF , anti- } \\
\text { VZV } \\
\text { lgM(+) } \\
\text { and VZV } \\
\text { DNA (+) }\end{array}$ & $\begin{array}{l}\text { Hemorrhage in right } \\
\text { intracerebellar }\end{array}$ & $\begin{array}{l}\text { Acyclovir } \\
\text { Methylprednisolone }\end{array}$ & Death \\
\hline 4 & $\begin{array}{l}\text { Jun } \\
\text { et al.2018 }\end{array}$ & Male,75 & No & $\begin{array}{l}\text { Herpes zoster, } \\
\text { headache, fever, } \\
\text { confusion, } \\
\text { hemiplegia }\end{array}$ & $\begin{array}{l}\text { CSF,VZV } \\
\operatorname{DNA}^{(+)}\end{array}$ & $\begin{array}{l}\text { Hemorrhage in the left } \\
\text { parietal lobe }\end{array}$ & Acyclovir & Death \\
\hline 5 & $\begin{array}{l}\text { Ganesh } \\
\text { et al.2018 }\end{array}$ & Female,79 & No & $\begin{array}{l}\text { Confusion, fever, } \\
\text { rash, paraplegia, } \\
\text { leg numbness }\end{array}$ & $\begin{array}{l}\text { CSF, VZV } \\
\text { DNA }(+)^{(+)}\end{array}$ & $\begin{array}{l}\text { Hemorrhage in the right } \\
\text { parietal lobe, occipital } \\
\text { horns of the lateral } \\
\text { ventricles }\end{array}$ & Not reported & $\begin{array}{l}\text { Not } \\
\text { reported }\end{array}$ \\
\hline 6 & $\begin{array}{l}\text { Maria } \\
\text { et al.2008 }\end{array}$ & Male,66 & No & $\begin{array}{l}\text { Headache, } \\
\text { nausea, vomit, } \\
\text { disorientation, } \\
\text { fever, neck } \\
\text { stiffness and } \\
\text { coma }\end{array}$ & $\begin{array}{l}\text { CSF, VZV } \\
\text { DNA and } \\
\text { IgG (+) } \\
\text { Serum, } \\
\text { VZV IgG } \\
(+)\end{array}$ & $\begin{array}{l}\text { Hemorrhage in the } \\
\text { temporal regions, } \\
\text { intraventricular and } \\
\text { subarachnoid } \\
\text { hemorrhage }\end{array}$ & $\begin{array}{l}\text { Acyclovir } \\
\text { Dexamethasone } \\
\text { Antibiotics }\end{array}$ & $\begin{array}{l}\text { Complete } \\
\text { recovery(0) }\end{array}$ \\
\hline
\end{tabular}

\section{Venous Sinus Thrombosis}

Three young men (20-30 years old) developed headache, fever, vomiting, papillary edema, and weakness in the left upper limb and left lower limb for two weeks after chickenpox infection. CSF was positive for anti-VZV IgG. MRV and MRI confirmed venous sinus thrombosis with diffuse cerebral edema. All patients had venous sinus thrombosis in the transverse sinus (TS). One of them had thrombosis in superior sagittal, bilateral transverse and right sigmoid sinuses. All patients improved completely with low-molecular-weight heparin and oral anticoagulants. Only one patient also received antiviral therapy and Another patient also had oral steroid.

The characteristics of the included cases were presented in Table 5.

Table 5

The characteristics of three venous sinus thrombosis cases

\begin{tabular}{|c|c|c|c|c|c|c|c|c|}
\hline Case & Reference & $\begin{array}{l}\text { Sex, } \\
\text { age(years) }\end{array}$ & Clinical features & $\begin{array}{l}\text { Diagnosis } \\
\text { test }\end{array}$ & $\begin{array}{l}\text { Time from } \\
\text { Herpes } \\
\text { zoster } \\
\text { infection }\end{array}$ & Affected brain region & Treatment & Outcome(mRS) \\
\hline 1 & $\begin{array}{l}\text { Anuradha } \\
\text { et al.2018 }\end{array}$ & Male,20 & $\begin{array}{l}\text { Chicken-pox, } \\
\text { headache, fever, } \\
\text { aphasic, left lateral } \\
\text { rectus palsy, } \\
\text { papilledema }\end{array}$ & $\begin{array}{l}\text { CSF and } \\
\text { serum ,anti- } \\
\text { VZV IgM(+) }\end{array}$ & 2 weeks & $\begin{array}{l}\text { Dural sinus } \\
\text { thrombosis(left } \\
\text { transverse, sigmoid } \\
\text { sinuses, and internal } \\
\text { jugular vein) }\end{array}$ & $\begin{array}{l}\text { Mannitol, } \\
\text { Anticoagulant } \\
\text { Steroids }\end{array}$ & $\begin{array}{l}\text { Complete } \\
\text { recovery }(0)\end{array}$ \\
\hline 2 & $\begin{array}{l}\text { Gayathri } \\
\text { et al.2016 }\end{array}$ & Male ,23 & $\begin{array}{l}\text { Rash, headache, } \\
\text { vomit, fever, } \\
\text { weakness of left } \\
\text { upper limb and left } \\
\text { lower limb }\end{array}$ & $\begin{array}{l}\text { CSF, anti- } \\
\text { VZV IgG(+) } \\
\text { and VZV } \\
\text { DNA(+) }\end{array}$ & 2 weeks & $\begin{array}{l}\text { Diffuse cerebral edema } \\
\text { Thrombosis in superior } \\
\text { sagittal, bilateral } \\
\text { transverse and right } \\
\text { sigmoid sinuses }\end{array}$ & $\begin{array}{l}\text { Acyclovir } \\
\text { Asprin } \\
\text { Anticoagulants }\end{array}$ & $\begin{array}{l}\text { Complete } \\
\text { recovery }(0)\end{array}$ \\
\hline 3 & $\begin{array}{l}\text { Sujay } \\
\text { et al.2012 }\end{array}$ & Male,30 & $\begin{array}{l}\text { Chicken-pox, } \\
\text { headache, vomit }\end{array}$ & $\begin{array}{l}\text { CSF, anti- } \\
\text { VZV IgG(+) }\end{array}$ & 15 days & $\begin{array}{l}\text { Diffuse cerebral edema } \\
\text { Thrombosis in superior } \\
\text { sagittal and right } \\
\text { transverse sinuses }\end{array}$ & $\begin{array}{l}\text { Heparin } \\
\text { Anticoagulants }\end{array}$ & $\begin{array}{l}\text { Complete } \\
\text { recovery }(0)\end{array}$ \\
\hline
\end{tabular}


Table 6

Comparison of demographics, Clinical features between ischemic stroke and intracerebal hemorrhage

\begin{tabular}{|c|c|c|c|c|}
\hline & & Ischemic stroke & Intracerebral hemorrhage & $P$ \\
\hline$n$ & & 25 & 6 & \\
\hline Age(years) & & 52 & 70.5 & 0.158 \\
\hline \multirow[t]{2}{*}{ Sex, $n(\%)$} & male & $18(72)$ & $3(50)$ & 0.358 \\
\hline & female & $7(28)$ & $3(50)$ & \\
\hline History of varicella zoster infection, $n(\%)$ & & $15(60)$ & $5(83.33)$ & 0.383 \\
\hline Headache, $n(\%)$ & & $9(36)$ & $4(66.67)$ & 0.208 \\
\hline Fever, $n(\%)$ & & $6(24)$ & $4(66.67)$ & 0.067 \\
\hline Cognitive impairment, $n(\%)$ & & $14(56)$ & $5(83.33)$ & 0.363 \\
\hline Hemiplegia, $n(\%)$ & & $8(32)$ & $4(66.67)$ & 0.174 \\
\hline Antiviral drug combined steroid, $n(\%)$ & & $15(n=24,62.50)$ & $2(n=5,40)$ & 0.370 \\
\hline
\end{tabular}

\section{Discussion}

In this study, we found that the incidence of ischemic stroke associated with VZV infection was high, accounting for about 73.53\%, while intracerebral hemorrhage or venous sinus thrombosis was relatively rare. The incidence in men was higher than in women in ischemia or venous sinus thrombosis groups. In terms of onset age, the middle-aged was common in the ischemic stroke group, the elderly in the hemorrhagic group, and the young in the venous sinus thrombosis group. Compared with other clinical symptoms, cognitive impairment was the most common either in the ischemic group or hemorrhagic group. The lesions after VZV-associated cerebral infarction or hemorrhage were mutifocal and was most common in the parietal lobe. If venous sinuses were involved, thrombosis was common in TS. Multiple stenosis of the anterior and posterior circulation vessels was found by DSA or MRA. Antiviral treatment may be useful in the ischemic group and anticoagulant therapy was essential in the venous sinus thrombosis group, while the role of glucocorticoids remained unclear in the treatment of VZV-associated stroke. Among the three groups, the patients with venous sinus thrombosis improved completely, however, the patients with intracerebral hemorrhage had poor prognosis.

There was no statistical significance in onset age or sex in our study despite the trend observed, which may need many large samples to get a credible conclusion. There was median time with $\mathbf{4 2 . 8}$ days from herpes zoster infection to the occurrence of neurologic symptoms in patients with ischemic stroke. Previous study has shown that there was usually a long delay (mean 4.1 months) ${ }^{[42]}$. The discrepancy was caused by different included samples, that is to say, children were included in other studies. Larissa et al. ${ }^{[43]}$ reported that the time from symptom onset to admission was 3.5 days after HSV infection. It meant there were some biological differences although both VZV and HSV were alpha-viruses. Clinical reactivation of HSV can occur repeatedly and mostly in the young, whereas clinical VZV reactivation typically occurs once per individual and predominantly in $25 \%$ of the elderly ${ }^{[44]}$. DNA replication occurs within $24 \mathrm{~h}$ for HSV, while VZV DNA replication can be seen as late as 5 days in human trigeminal ganglionic explants [45]. There may partly contribute to the different latency between HSV and VZV infection.

The main clinical manifestations of patients were cognitive impairment, followed by headache, hemiplegia whether in the ischemic group or in the hemorrhagic group, which may be related with cerebral damaged locations caused by vasculopathy associated with VZV infection. In the two groups, the lesions were multiple and involved in the parietal, frontal or temporal lobes. It didn't accord with the characteristics of cerebrovascular diseases induced by atherosclerosis, and was more prone to angioinflammatory lesions. In this study, we found all the three men had have thrombosis in TS. As we know the superior sagittal sinus was the major anatomical site of sinus thrombosis, followed by TS. However, hypoplasia or aplasia of TS is a common anatomic variation ${ }^{[46,47]}$.This made some people with the variation more prone to sinus thrombosis in the presence of certain risk factors, such as inflammation, infection or hypercoagulability.

Ischemic lesions associated with VZV encephalitis were common while herpes simplex encephalitis (HSE) is a hemorrhagic necrotizing inflammatory process, indicating different mechanisms of vasculopathy after infection of the two viruses. It has been found that direct cytolytic virus replication and indirect immune-mediated processes are responsible for neurons, glial and axonal damage in the pathologic course of HSE ${ }^{[48]}$. But after VZV reactivation, the virus spreads axially along the trigeminal nerve and other cerebral ganglion where it is long dormant, to infect the arterial adventitia and then extend transmurally through the whole artery well. The pathological manifestations were disruption of the internal elastic lamina,hyperplastic intima, decreased medial smooth muscle cells and inflammatory cell infiltration. It was further confirmed by Gilden et al., and they found the existence of VZV particles in the cerebral vessels through anatomical and pathological examination of the whole brain in the patients who died after VZV infection ${ }^{[49]}$. To some extent, it explained the mechanism of vasculitis response after VZV infection. In this research, multiple stenosis of the anterior and posterior circulation vessels was examined by DSA or MRA. Lesions in large vessels were common, followed by small vessels in ischemic group and multiple beaded stenosis were showed in hemorrhagic group. All three patients with venous sinus thrombosis had thrombus in two or more vessels. This angiopathic characteristics were more consistent with vasoinflammatory lesions. 
Katchanov et al. detected a direct enhancement of the arterial wall in their patients with VZV vasculopathy ${ }^{\text {[22] }}$. The detection of contrast material within the vessel wall on enhanced T1-weighted images was considered to be a direct radiological sign of vasculitis and visualizes the inflammatory process in the vessel wall ${ }^{[50]}$. High-resolution MRI (HRMR) ${ }^{[51]}$ revealed vessel wall thickening and enhancement in patients with VZV vascular disease, and after treatment showed improvement of stenosis. Swartz et al. evaluated intracranial vascular lesions induced by atherosclerotic disease or CNS inflammatory disease. They found there was focal, eccentric vessel wall enhancement for the former, However, there was diffuse, concentric vessel wall enhancement for the CNS inflammatory diseases ${ }^{[52]}$.

Specificity of VZV DNA is $97 \%$, enabling rapid diagnosis and early treatment. But VZV DNA in the CSF cannot be detected 14-50 days after infection because of the incubation period. The value of anti-VZV IgG antibody is greater than that of VZV DNA in chronic cases ${ }^{[53]}$. The diagnosis of vascular diseases related with VZV can only be ruled out when both CSF VZV DNA and anti-VZV IgG antibodies are negative. In our study, the diagnosis was confirmed by detecting anti-VZV IgG antibody or VZV DNA in CSF. In particular, one patient was diagnosed by viral culture.

In ischemic stroke group, early intravenous acyclovir was given to all patients whose treatments were referred to, and most of them improved. It suggested antiviral treatment may be useful. There was no significant difference in either mortality or favorable prognosis between antiviral medication therapy alone and antiviral drug combined with steroid, suggesting the role of glucocorticoids in the treatment of VZV-associated ischemic stroke remains unclear. Only one patient received anti-platelet treatment besides the usage of antiviral and steroid and recovered well. In a randomized clinical trial of tuberculous meningitis, aspirin reduced mortality by $19 \%{ }^{[54]}$. But the efficacy of anti-platelet drugs in acute ischemic events of infection-associated stroke was unknown. Prospective, multicenter and randomized controlled trials are currently required to evaluate the efficacy of antiplatelet drugs and/or steroid therapy in ischemic stroke induced by VZV infection.

More than half of the patients with intracerebral hemorrhage had poor prognosis. The reasons may be related to advanced age of onset, brain stem compression, cerebellar involvement and severe tissue edema. The three young male patients with venous sinus thrombosis in our study had good prognosis after anticoagulant therapy, indicating the necessity of anticoagulation therapy.

\section{Conclusion}

Cerebral diseases associated with VZV encephalitis is not uncommon. When the patient represents with some neurological symptoms within 2 months after VZV infection, and multiple lesions probably induced by vasculitis showed in neuroimaging, cerebral complications related with VZV infection should be considered even though the existence of some vascular risk factors for atherosclerosis. HRMR is an alternative method to distinguish the nature of vascular lesions. Ischemic stroke related with VZV encephalitis is common and mainly affects the middle-aged. In general, the young patients with venous sinus thrombosis improve completely, however, the old patients with intracerebral hemorrhage have poor prognosis.

We acknowledge several limitations to our study. A publishing bias for severe cerebral vascular diseases after VZV infection must be anticipated. There were only six cases of intracerebral hemorrhage and three cases of venous sinus thrombosis. More large samples and evidence-based medical studies are needed to clarify informative conclusions.

\section{List Of Abbreviations}

\section{VZV}

varicella-zoster virus; HSV:herpes simplex virus; CNS:central nervous system; CT:computed tomography; MRI:magnetic resonance imaging; MRA:magnetic resonance angiography; MRV magnetic resonance venography; DSA:digital subtraction angiography; CSF:cerebrospinal fluid; mRS:modified Rankin scale; TS:transverse sinus; HSE:herpes simplex encephalitis; HRMR:high-resolution magnetic resonance imaging

\section{Declarations}

\section{Acknowledgements}

Not applicable.

\section{Authors' contributions}

The final manuscript was read and approved by all authors. Hangfei Wu performed the experiments, analyzed the data, generated the figures, and drafted the manuscript. Ruoru Wang analyzed the data, generated the figures, and drafted the manuscript. Yuanyuan Li and Xu Sun analyzed the data. Jiasi Li and Xiaoying Bi designed and monitored this study and edited the manuscript.

\section{Availability of data and materials}

All data are fully available without restriction.

\section{Funding}

This study was supported by the National Nature Science Foundation of China (NO. 81971242). 


\section{Ethics approval and consent to participate}

Not applicable.

\section{Consent for publication}

Not applicable.

\section{Competing interests}

The author(s) declared no potential conflicts of interest with respect to the research, authorship, and/or publication of this article.

\section{References}

1. Gilden DH, Vafai A, Shtram Y, Becker Y, Devlin M, Wellish M. Varicella-zoster virus DNA in human sensory ganglia. Nature. 1983;306:478-80.

2. Tyler KL. Acute Viral Encephalitis. N Engl J Med. 2018;379:557-66.

3. Gilden DH, Kleinschmidt-DeMasters BK, LaGuardia JJ, Mahalingam R, Cohrs RJ. Neurologic complications of the reactivation of varicella-zoster virus. N Engl J Med. 2000;342:635-45.

4. Johnson R, Milbourn PE. Central nervous system manifestations of chickenpox. Can Med Assoc J. 1970;102:831-4.

5. Yawn BP, Wollan PC, Nagel MA, Gilden D. Risk of Stroke and Myocardial Infarction After Herpes Zoster in Older Adults in a US Community Population. Mayo Clin Proc. 2016;91:33-44.

6. Fugate JE, Lyons JL, Thakur KT, Smith BR, Hedley-Whyte ET, Mateen FJ. Infectious causes of stroke. Lancet Infect Dis. 2014;14:869-80.

7. Breuer J, Pacou M, Gautier A, Brown MM. Herpes zoster as a risk factor for stroke and TIA: a retrospective cohort study in the UK. Neurology. 2014;83:e27-33

8. Venkatesan A, Tunkel AR, Bloch KC, et al. Case definitions, diagnostic algorithms, and priorities in encephalitis: consensus statement of the international encephalitis consortium. Clin Infect Dis. 2013;57:1114-28.

9. Sulter G, Steen C, De Keyser J. Use of the Barthel index and modified Rankin scale in acute stroke trials. Stroke. 1999;30:1538-41.

10. Powell DR 2nd, Patel S, Franco-Paredes C. Varicella-Zoster Virus Vasculopathy: The Growing Association Between Herpes Zoster and Strokes. Am J Med Sci. 2015;350:243-5.

11. Venhovens J, Stelten B, Feyen BF, van Dijk G, Meulstee J. Ischemic Stroke as a Complication of Varicella Zoster Encephalitis: A Case Report With Detailed EEG Discussion. Clin EEG Neurosci. 2014;45:310-4.

12. Teodoro T, Nagel MA, Geraldes R, et al. Biopsy-negative, varicella zoster virus (VZV)-positive giant cell arteritis, zoster, VZV encephalitis and ischemic optic neuropathy, all in one. J Neurol Sci. 2014;343:195-7.

13. Chiang F, Panyaping T, Tedesqui G, Sossa D, Costa Leite C, Castillo M. Varicella zoster CNS vascular complications. A report of four cases and literature review. Neuroradiol J. 2014;27:327-33.

14. Calabria F, Zappini F, Vattemi G, Tinazzi M. Pearls. \& Oy-sters: an unusual case of varicella-zoster virus cerebellitis and vasculopathy. Neurology. 2014;82:e14-5.

15. Silver B, Nagel MA, Mahalingam R, Cohrs R, Schmid DS, Gilden D. Varicella zoster virus vasculopathy: a treatable form of rapidly progressive multiinfarct dementia after 2 years' duration. J Neurol Sci. 2012;323:245-7.

16. Miyazaki Y, Riku Y, Goto Y, Mano K, Yoshida M, Hashizume Y. VZV vasculopathy associated with myelo-radiculoganglio-meningo-encephalitis: an autopsy case of an immunocompetent 66-year-old male. J Neurol Sci. 2008;275:42-5.

17. Outteryck O, Senechal O, Berteloot D, Delalande I, Mounier-Vehier F. [Cerebral vasculitis secondary to Varicella-Zoster virus infection]. Rev Neurol (Paris). 2005;161:836-9

18. Lopez-Gomez M, Lopez-Ruz MA, Jimenez-Alonso JF. [Cerebral infarction due to varicella-zoster virus in a patient with HIV infection]. Enferm Infecc Microbiol Clin. 2003;21:532-3.

19. Ahmad NM, Boruchoff SE. Multiple cerebral infarcts due to varicella-zoster virus large-vessel vasculopathy in an immunocompetent adult without skin involvement. Clin Infect Dis. 2003;37:e16-8.

20. Ratchford JN, Costello K, Reich DS, Calabresi PA. Varicella-zoster virus encephalitis and vasculopathy in a patient treated with fingolimod. Neurology. 2013;81:306.

21. McKelvie PA, Collins S, Thyagarajan D, Trost N, Sheorey H, Byrne E. Meningoencephalomyelitis with vasculitis due to varicella zoster virus: a case report and review of the literature. Pathology. 2002;34:88-93.

22. Katchanov J, Siebert E, Klingebiel R, Endres M. Infectious vasculopathy of intracranial large-and medium-sized vessels in neurological intensive care unit: a clinico-radiological study. Neurocrit Care. 2010;12:369-74.

23. Gilden DH, Lipton HL, Wolf JS, et al. Two patients with unusual forms of varicella-zoster virus vasculopathy. N Engl J Med. 2002;347:1500-3.

24. Salazar R, Russman AN, Nagel MA, et al. Varicella zoster virus ischemic optic neuropathy and subclinical temporal artery involvement. Arch Neurol. 2011;68:517-20. 
25. Kronenberg A, Schupbach R, Schuknecht B, et al. Multifocal vasculopathy due to Varicella-Zoster Virus (VZV): serial analysis of VZV DNA and intrathecal synthesis of VZV antibody in cerebrospinal fluid. Clin Infect Dis. 2002;35:330-3.

26. Russman AN, Lederman RJ, Calabrese LH, Embi PJ, Forghani B, Gilden DH. Multifocal varicella-zoster virus vasculopathy without rash. Arch Neurol. 2003;60:1607-9.

27. Saraya T, Shimura C, Wada H, Aoshima M, Goto H. Evidence for vascular spread of varicella zoster-associated vasculopathy. Ann Intern Med. 2006;144:535-7.

28. Vibha D, Prabhakar S, Khurana D, Khandelwal N. Varicella zoster vasculopathy presenting as lateral medullary syndrome. J Neurovirol. 2012;18:53840.

29. Ortiz GA, Koch S, Forteza A, Romano J. Ramsay hunt syndrome followed by multifocal vasculopathy and posterior circulation strokes. Neurology. 2008;70:1049-51.

30. Kalita J, Das M, Misra UK. Herpes Zoster Ophthalmicus Leading to Middle Cerebral Artery Infarction: A Case Report. International Journal of Angiology. 2004.

31. Mareedu J, Hanumaiah RG, Hale E, Habte-Gabr E. Varicella zoster vasculopathy. J Int Assoc Physicians AIDS Care (Chic). 2011;10:144-5.

32. Tavazzi E, Minoli L, Ferrante P, et al. Varicella zoster virus meningo-encephalo-myelitis in an immunocompetent patient. Neurol Sci. 2008;29:279-83.

33. Gonzalez-Suarez I, Fuentes-Gimeno B, Ruiz-Ares G, Martinez-Sanchez P, Diez-Tejedor E. Varicella-zoster virus vasculopathy. A review description of a new case with multifocal brain hemorrhage. J Neurol Sci. 2014;338:34-8.

34. Baek W, Lee SG, Kim YS, Kim JH, Jun JB, Kim HY. Fatal varicella-zoster virus vasculopathy associated with adalimumab therapy. Arch Neurol. 2012;69:1193-6.

35. Matsuo K, Uozumi Y, Miyamoto H, Tatsumi S, Kohmura E. Varicella-zoster vasculitis presenting with cerebellar hemorrhage. J Stroke Cerebrovasc Dis. 2015;24:e153-5.

36. Takeshita J, Nomura E, Takemaru M, Himeno T, Shimoe Y, Kuriyama M. [Rapidly deteriorated lobar intracerebral hemorrhages: possible association of varicella zoster virus-vasculopathy]. Rinsho Shinkeigaku. 2018;58:245-8.

37. Ganesh A, Kashani N, Bal SS, Jenkins J, Yeung MMC. Teaching Neurolmages: Varicella-zoster virus-related hemorrhagic encephalomyelitis. Neurology. 2018;90:e1360-1.

38. Mpaka M, Karantanas AH, Zakynthinos E. Atypical presentation of varicella-zoster virus encephalitis in an immunocompetent adult. Heart Lung. 2008;37:61-6.

39. Mehta A, Arora A, Sharma M, Malik R, Porwal YC. Hemorrhagic Stroke and Cerebral Venous Thrombosis: Rare Neurological Sequelae of Chickenpox Infection. Ann Indian Acad Neurol. 2018;21:228-32.

40. Gayathri K, Ramalingam PK, Santhakumar R, et al. Cerebral Sinus Venous Thrombosis as a Rare Complication of Primary Varicella Zoster Virus Infection. J Assoc Physicians India. 2016;64:74-6.

41. Sada S, Kammineni A, Kanikannan MA, Afshan J. Cerebral sinus venous thrombosis: a rare complication of primary Varicella zoster virus. Neurol India. 2012;60:645-6.

42. Nagel MA, Cohrs RJ, Mahalingam R, et al. The varicella zoster virus vasculopathies: clinical, CSF, imaging, and virologic features. Neurology. 2008;70:853-60.

43. Hauer L, Pikija S, Schulte EC, Sztriha LK, Nardone R, Sellner J. Cerebrovascular manifestations of herpes simplex virus infection of the central nervous system: a systematic review. J Neuroinflammation. 2019;16:19.

44. Mitchell BM, Bloom DC, Cohrs RJ, Gilden DH, Kennedy PG. Herpes simplex virus-1 and varicella-zoster virus latency in ganglia. J Neurovirol. 2003;9:194-204.

45. Azarkh Y, Bos N, Gilden D, Cohrs RJ. Human trigeminal ganglionic explants as a model to study alphaherpesvirus reactivation. J Neurovirol. 2012;18:456-61.

46. Bousser MG, Ferro JM. Cerebral venous thrombosis: an update. Lancet Neurol. 2007;6:162-70.

47. Damak M, Crassard I, Wolff V, Bousser MG. Isolated lateral sinus thrombosis: a series of 62 patients. Stroke. 2009;40:476-81.

48. Piret J, Boivin G. Innate immune response during herpes simplex virus encephalitis and development of immunomodulatory strategies. Rev Med Virol. 2015;25:300-19.

49. Gilden DH, Kleinschmidt-DeMasters BK, Wellish M, Hedley-Whyte ET, Rentier B, Mahalingam R. Varicella zoster virus, a cause of waxing and waning vasculitis: the New England Journal of Medicine case 5-1995 revisited. Neurology. 1996;47:1441-6.

50. Kuker W, Gaertner S, Nagele T, et al. Vessel wall contrast enhancement: a diagnostic sign of cerebral vasculitis. Cerebrovasc Dis. 2008;26:23-9.

51. Cheng-Ching E, Jones S, Hui FK, et al. High-resolution MRI vessel wall imaging in varicella zoster virus vasculopathy. J Neurol Sci. 2015;351:168-73.

52. Swartz RH, Bhuta SS, Farb RI, et al. Intracranial arterial wall imaging using high-resolution 3-tesla contrast-enhanced MRI. Neurology. 2009;72:627-34.

53. Nagel MA, Forghani B, Mahalingam R, et al. The value of detecting anti-VZV IgG antibody in CSF to diagnose VZV vasculopathy. Neurology. 2007;68:1069-73.

54. Misra UK, Kalita J, Nair PP. Role of aspirin in tuberculous meningitis: a randomized open label placebo controlled trial. J Neurol Sci. 2010;293:12-7. 